\title{
Neue Lithium-Glaskeramik steht für ein hohes Maß an Sicherheit
}

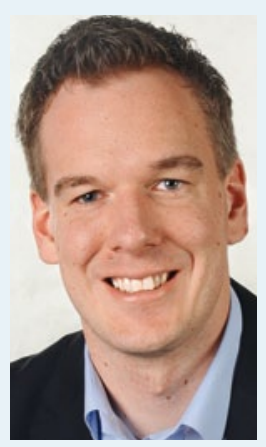

M. Gödicker
Dipl.-Ing. Michael

Gödiker (Fachbe-

reichsleiter VITAF \& E,

Bad Säckingen) war

maßgebend an der

Entwicklung von VITA

SUPRINITY, der neuen

zirkondioxidverstärkten

Lithiumsilikat-Glaskeramik (ZLS), beteiligt. Im Interview erläutert er die Besonderheiten des neuartigen CAD/CAM-

Werkstoffs, beschreibt seine speziellen mechanischen Eigenschaften und berichtet von den Ergebnissen aus den Leistungstests.

? Herr Gödiker, was waren die Ziele der Entwicklung von VITA SUPRINITY und was ist das Neue an diesem Werkstoff?

Michael Gödiker: Ziel war die Realisierung eines hochfesten, zuverlässigen, ästhetischen Materials, das sich leicht verarbeiten lässt. Herausgekommen ist die neue Materialklasse ZLS mit einer zirkondioxidangereicherten Glasphase und feinkristallinem Gefüge.

? Welche Ergebnisse erreicht VITA SUPRINITY im Leistungstest - insbesondere in Bezug auf die mechanische Festigkeit und die Bruchlast?

Gödiker: Wir geben zunächst eine 3-Punkt-Biegefestigkeit von $420 \mathrm{MPa}$ an. Dieser Wert beschreibt die mittlere Festigkeit, die im Rahmen zahlreicher Char-

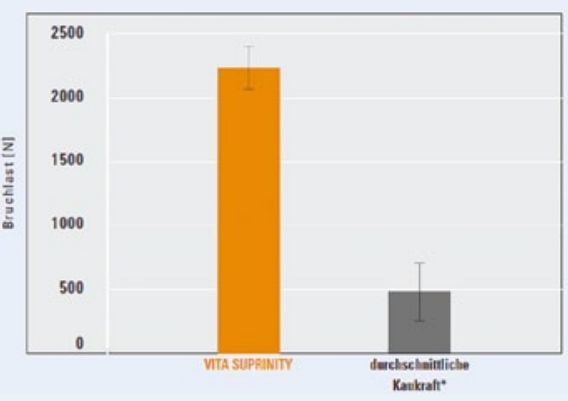

VITA SUPRINITY hält einer Belastung von ca. $2262 \mathrm{~N}$ stand.

Quelle: Interne Untersuchung, VITA F \&E

genprüfungen mit über 800 Einzelwerten bei reduziertem Präparationsaufwand ermittelt wurde. Bei Präparation gemäß Norm (ISO 6872) werden in der 3- oder 4-Punkt-Biegeprüfung auch höhere Mittelwerte im Bereich von 500 MPa erreicht. Um die klinische Bewährung zu simulieren, haben wir zudem statische und dynamische Bruchlasttests durchgeführt. Der ermittelte statische Bruchlastwert liegt bei ca. 2262 Newton (N) und damit weit über der mittleren maximalen Kaukraft von etwa 490 N. Erstaunlich war auch das Ergebnis des dynamischen Belastungstests mit einer Überlebensrate von $100 \%$ bei adhäsiv befestigten VITA SUPRINITY-Kronen, belastet mit einer Kraft von $1200 \mathrm{~N}$ über 1,2 Mio. Zyklen.

? Was bedeutet die ermittelte hohe Belastbarkeit für den klinischen Einsatz und welche Vorteile ergeben sich daraus für den Praxis- und Laboranwender?
Gödiker: Sie steht für ein hohes Maß an Sicherheit hinsichtlich der Lebensdauer der Versorgungen und ermöglicht in der praktischen Anwendung beispielsweise reduzierte Wandstärken gegenüber Restaurationen aus Feldspatkeramik.

? VITA SUPRINITY wird seit einiger Zeit auch in der klinischen Anwendung erprobt. Welche Rückmeldung erhalten Sie von Praxen und Laboren? Gödiker: Generell wurde VITA SUPRINITY sehr positiv beurteilt. Besonders hervorgehoben wurden die ästhetischen Eigenschaften sowie die herausragende Schleifund Polierbarkeit. Auch die zugehörige Verblendkeramik VITA VM11 wurde bezüglich der Verarbeitung sowie der erzielbaren Ästhetik als sehr gut beurteilt.

? Wenn Sie den Blick etwas in die Zukunft richten, wo sehen Sie die neue Glaskeramikgeneration in einigen Jahren?

Gödiker: Sie besitzt das Potenzial, zur standardmäßigen Kronen- und Brückenversorgung im Front und Seitenzahnbereich zu werden. Für die nahe Zukunft sind ein final kristallisierter Block und eine Pressvariant ein Vorbereitung. Langfristig werden Produkteigenschaften sowie das daraus resultierende Angebot stetig weiter entwickelt.

Nach einer Pressemitteilung der

VITA Zahnfabrik H. Rauter GmbH \& Co. KG, Bad Säckingen

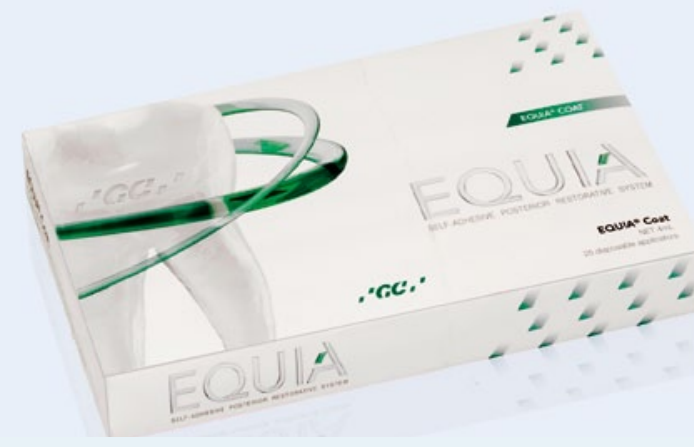

Mit EQUIA bietet GC ein 2-stufiges Füllungskonzept an, das einer für das Kind und den Zahnarzt möglichst entspannten und kurzen Behandlung entgegenkommt. Klinische Studien und die Erfahrung aus der Praxis belegen die große Haltbarkeit des Systems, das eine hochviskose Glasionomerkomponente (EQUIA Fil) mit einem nano-gefüllten, lichthärtenden Kompositlack (EQUIA Coat) vereint. Eine zusätzliche kariesprotektive Wirkung spricht ebenfalls für den Einsatz von EQUIA im Milchzahngebiss. Das Füllungs- konzept bietet eine kurze Verarbeitungszeit, wirkt kariespräventiv und ist gemäß der Gebrauchsanweisung des Herstellers unter anderem bei Restaurationen der Klasse I, unbelasteten Restaurationen der Klasse II und kaudruckbelasteten Restaurationen der Klasse II (sofern der Isthmus weniger als die Hälfte des Interkuspidalraumes beträgt) anwendbar und über die GKV abrechnungsfähig.

Nach einer Pressemitteilung der GC Germany GmbH, Bad Homburg Internet: www.germany.gceurope.com 
Akustikstudie

Innovatives Spray macht besonders leise

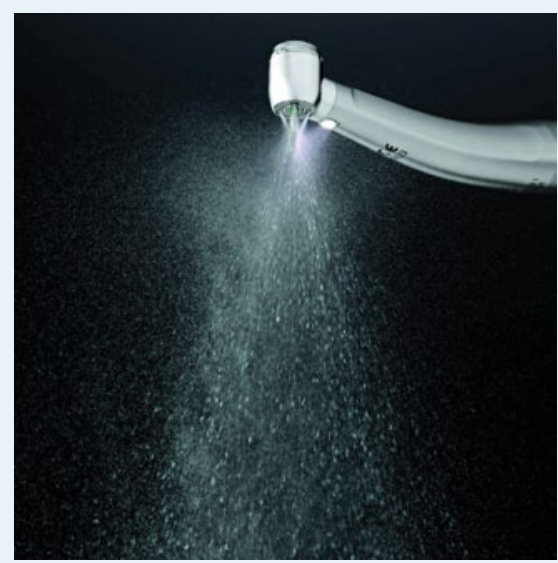

Die Lautstärke von Dental-Turbinen wird nicht allein durch Lauf- und Bohrgeräusche verursacht, sondern auch durch das Spray. Akustiker testeten Turbinen unterschiedlichster Provenienz im Hinblick auf Schalldruck, Lautheit und Schärfe miteinander. Dabei wurde deutlich, dass der Lärm nicht allein durch Lauf- und Bohrgeräusche entsteht, sondern auch durch das Spray, das zur Kühlung eingesetzt wird. Die meisten Turbinen waren im Test signifikant lauter, wenn sie mit Spray betrieben wurden, teilweise bis zu $10 \mathrm{~dB}(\mathrm{~A})$ - was einer Verdopplung der Lärmbelastung entspricht. Die geringsten Abweichungen im Schalldruck gab es bei den beiden Sirona-Turbinen T1 Control S und T2 Boost S, die bei Nutzung des Sprays nur um 2,2 bzw. 0,2 dB(A) lauter waren als ohne. Der Grund: Sirona hat 2013 eine neue Generation von Turbinen eingeführt, die über ein innovatives 4-Düsen-Spray verfügt. Dank dieser Innovation waren die Sirona-Turbinen im Vergleich unter Einsatz von Spray leiser als die anderen Test-Kandidaten. Um anschaulich vorzuführen, wie leise die Turbinen von Sirona sind, hat das Unternehmen eine SoundCheck-App entwickelt. Ziel des Spiels ist es, Bohrgeräusche sowie Geräusche aus der Natur der Lautstärke nach zu ordnen. Teilnehmer an dem Spiel können Sennheiser-Kopfhörer HD 600 Stereo gewinnen. Im App Store ist der „Sirona SoundCheck“ verfügbar unter: https://itunes.apple.com/de/app/sironasoundcheck/id806229729?mt=8

Nach einer Pressemitteilung der Sirona Dental GmbH, A-Wals bei Salzburg Internet: www.sirona.com

\section{Hygiene \\ Steril ohne Aufpreis}

Ab 1.4.2014 stellt Komet sein zahnärztliches Standardsortiment auf eine serienmäßig sterile Auslieferung in Deutschland und Österreich ohne Aufpreis um. Der Service startet mit der klassischen BlisterStandardverpackung und wird sukzessive bis Herbst 2014 auf das zahnärztliche Standardsortiment - in Summe $2000 \mathrm{Ar}$ tikel - übertragen. Komet hat seine Firmenzentrale in Lemgo um ein $2000 \mathrm{~m}^{2}$ großes Logistikgebäude erweitert. Markantester Bestandteil dieses Gebäudes ist der $250 \mathrm{~m}^{2}$ große Reinraum. In dieser Einrichtung können jährlich von eigens geschultem Fachpersonal über 10 Millionen Instrumente unter Reinraumbedingungen verpackt werden. Anschließend erfolgt die Gammasterilisation bei einem Fachunternehmen. Im Reinraum werden Produkte aus den Geschäftsbereichen Medical und Dental verpackt.

Mit dem neuen Zero-Standard entfällt die Aufbereitung vor dem 1. Einsatz am Patienten, die bewährte Komet-Qualität

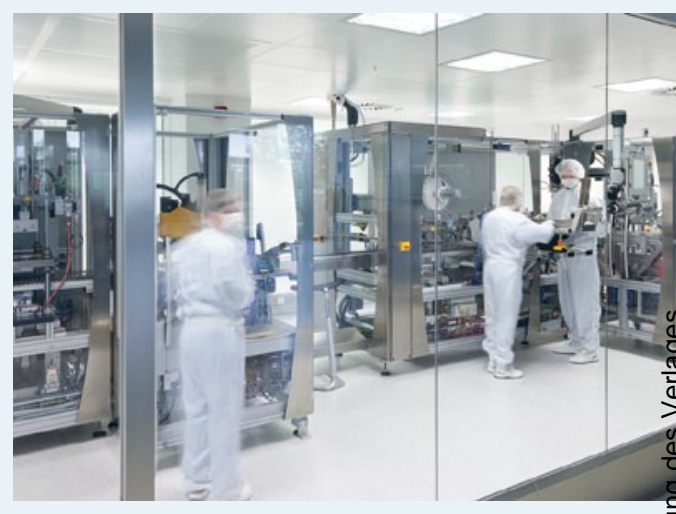

bleibt selbstverständlich erhalten. Keine Frage also: In Zukunft spart eine Praxis mit dem kostenlosen Zero-Standard Zeit und Geld. Weiterführende Informationen sind auf der Komet-Microsite (www. komet-steril.de) zu finden.

Nach einer Pressemitteilung der Komet Dental Gebr. Brasseler GmbH \& Co KG, Lemgo

Internet: www.kometdental.de

\section{Prophylaxe \\ Zahnpasta mit 10-fach-Wirksystem kostenlos testen}

Signal hat sich bei der Entwicklung der neuen Wirkformel für die Signal Expert Protection von Zahnseide und Mundspülung inspirieren lassen. Die Multifunktionszahnpasta mit ihrer enthaltenen Kombination aus Mikrogranulaten, Hydroxyapatit, Fluorid und Zinkzitrat fördert die Mund- und Zahngesund-

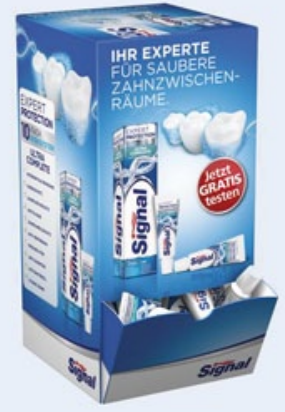
dung sowie Bakterien. Ab sofort können deutsche Zahnarztpraxen kostenlos pro Praxis bis zu 2 TresenAufsteller mit je 100 Gratis Zahnpasta-Probiertuben Signal Expert Protection für ihre Patienten bestellen (solange Vorrat reicht). Senden Sie einfach eine E-Mail mit der genauen Praxisanschrift an heit mit dem 10-fach-Wirksystem - für die professionelle Zahnpflege zu Hause. Sie bietet mit ihren Mikrogranulaten im Vergleich zu einer Zahnpasta ohne Mikrogranulate eine bessere interdentale Reinigung, stärkt mit Fluorid und Hydroxyapatitden Zahnschmelz, schützt die Zähne 18 Stunden vor Plaqueneubildung, remineralisiert den Zahnschmelz, entfernt schonend Verfärbungen und stärkt das Zahnfleisch. Die antibakterielle Wirkung von Zink erfrischt den Atem, bekämpft die Zahnsteinneubil-

signal@directpunkt.de.

Zusätzlich erhält das Praxisteam je eine Zahnpasta Signal Expert Protection Ultra Complete, Brilliant White und Pure Freshness $(75 \mathrm{ml}$ ) sowie 2 Terminblöcke mit Rabattcoupons. Mit diesen bekommen Patienten vom 01.04.-30.08.2014 in allen deutschen dm-Märkten beim Kauf einer Signal Expert Protection einen Rabatt in Höhe von $0,50 €$.

Nach einer Pressemitteilung der Unilever Deutschland GmbH, Hamburg

Internet: www.signal-zahnpflege.de 
Neues Online-Tool

\section{Zementauswahl per Mausklick}

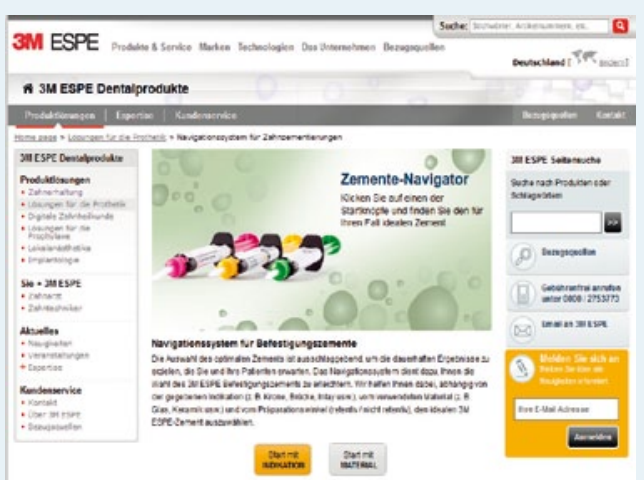

Dank der großen Anzahl an verfügbaren Restaurationsmaterialien ist es heute möglich, für jeden Patienten und jede Indikation die passende Versorgung herzustellen. Für einen optimalen Verbund zwischen Zahn und Restauration sorgen unterschiedliche Befestigungszemente, die indikationsund materialgerecht auszuwählen sind. Um Anwender bei der Zementauswahl optimal zu unterstützen, hat 3M ESPE einen Online-Navigator entwickelt, mit dem sich mit nur wenigen Klicks das ideal geeignete Befestigungsmaterial ermitteln lässt - für effiziente Workflows und beste Resultate.
Durch diese Auswahl wird sichergestellt, dass für jede Indikation, jede Art der Präparation und jedes Restaurationsmaterial ein optimal geeigneter Zement von 3M ESPE zur Verfügung steht. Die verfügbaren Zemente unterscheiden sich vor allem hinsichtlich der Art des erzielten Verbundes zwischen Zahnsubstanz/Abutment und Versorgung (mikromechanische Retention versus chemischer Verbund), der ästhetischen Eigenschaften und der Einfachheit der Anwendung. Der Online-Navigator hilft dabei, stets das Befestigungsmaterial auszuwählen, das für die jeweilige Situation die optimale Kombination von Eigenschaften bietet. Unter www.3MESPE.de/Zementenavigator werden die vorliegende Indikation und das verwendete Restaurationsmaterial in beliebiger Reihenfolge ausgewählt. Daraufhin wird das empfohlene Befestigungsmaterial von 3M ESPE angezeigt auf Wunsch gleich mit Detailinformationen zum Produkt sowie einer Verarbeitungsanleitung.

Nach einer Pressemitteilung der 3M Deutschland GmbH, Standort Seefeld

\section{Round-Table-Meeting der Carestream Dental Premium Referenz Partner}

Gut 20 Carestream DVT-Anwender aus den wichtigsten dentalen Disziplinen trafen sich vom 14.-16.03. auf der hochkarätig besetzten Veranstaltung in München mit Unternehmensvertretern, um im Westin Grand Hotel neueste digitale Techniken und dentale Fachthemen von CAD/CAM über Entwicklungen und Anforderungen im DVT-Bereich bis hin zu den Möglichkeiten der Außendarstellung in den Medien zu diskutieren. Ziel war es, über einen intensiven - und dabei vollkommen offenen Austausch zwischen Anbietern und Anwendern - ein direktes Feedback zu erhalten, sowie zukünftige Maßnahmen und Entwicklungsschritte noch besser als

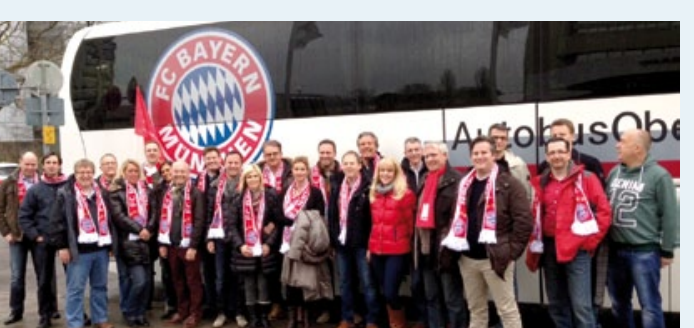

bisher auf die Bedürfnisse des Marktes zuzuschneiden. Zum Abschluss wartete mit einem gemeinsamen Besuch des Bundesliga Spitzenspiels zwischen FC Bayern München und Bayer Leverkusen ein weiteres Highlight auf die Besucher. Dies wird nicht die letzte Veranstaltung dieser Art gewesen sein. Frank Bartsch, Trade Marketing Manger bei Carestream Dental: „Die positive Resonanz hat uns gezeigt, dass wir mit diesem Round-Table-Meeting den richtigen Weg beschritten haben - wenn wir auch fast schon zu wenig Zeit hatten. Nur der intensive, gerne auch kritische, aber immer konstruktive Austausch mit den Anwendern garantiert, dass wir auch in der Zukunft der Innovationsführer bei den bildgebenden Verfahren sein werden“.

Nach einer Pressemitteilung der CarestreamHealth Deutschland GmbH, Stuttgart Internet: www.carestreamdental.de des neuen Logos

\section{FLEMMING}

Ihre Dental-Experten vor Ort

Seit Anfang des Jahres präsentiert sich Flemming Dental mit einem komplett neuen Markenauftritt. Das neue Design setzt frische Akzente durch ein helles Grün in Kombination mit dem bisher bekannten Blau der Marke. Das Unternehmen bietet an seinen rund 40 Standorten in Deutschland beste handwerkliche Qualität - ausschließlich „Made in Germany“. Der funktionell und ästhetisch hochwertig gefertigte Zahnersatz wird den Kunden zu einem fairen Preis-Leistungs-Verhältnis angeboten. Kontinuierlich werden im Dental-Partner-Technologiezentrum (DPT) in Leipzig neue Materialien und Verfahren getestet, um innovative Produkte und Techniken zu entwickeln. Parallel dazu werden den Zahnarztkunden Mehrwertleistungen durch das Schwesterunternehmen Dental Partner Care (DPC) angeboten. Hierzu gehören günstige Einkaufskonditionen für den Praxisbedarf, Unterstützung im Praxismarketing, eine Abrechnungsberatung, Technologieschulungen und Fortbildungsveranstaltungen. Der moderne Internetauftritt unter www.flemmingdental.de lädt ein, sich mit wenigen „Klicks“ auf der übersichtlichen Seite umzuschauen und sich einen Überblick über das komplexe Leistungsspektrum zu verschaffen. Jede Niederlassung hat ihre eigene Webseite mit einer Laborvorstellung, einem Veranstaltungskalender, Patienteninformationen und Neuigkeiten, die den individuellen Charakter des einzelnen Standortes widerspiegelt.

Einer der großen Vorzüge aus Kundensicht ist die Verfügbarkeit des Labors direkt vor Ort und der ständige enge Dialog. Im neuen Slogan wird dies aufgegriffen: „Flemming - Ihre Dental-Experten vor Ort“.

Nach einer Presseinformation der Flemming Dental Service GmbH, Hamburg Internet: www.flemming-dental.de 


\section{Neues Glasionomer-Füllungsmaterial in Applikationskapseln}

IonoStar Molar ist ein neu entwickeltes Glasionomer-Füllungsmaterial in der neuen VOCO-Applikationskapsel. Das Material wird ohne Conditioner und Adhäsiv angewendet. Es lässt sich sofort nach dem Einbringen modellieren und härtet nach $4 \mathrm{~min}$ aus. Seine lang anhaltende hohe Fluoridabgabe wirkt postoperativen Sensitivitäten entgegen. Zusammen mit Easy Glaze, dem nanogefüllten Lack zur Oberflächenversiegelung, lassen sich mit IonoStar Molar ebenso ästheti- sche wie haltbare Restaurationen schnell und einfach herstellen. IonoStar Molar eignet sich für Füllungen von nicht okklusionstragenden Kavitäten der Klasse I, semipermanente Füllungen von Kavitäten der Klasse I und II, Füllungen von Zahnhalsläsionen, Klasse-V-Kavitäten, Behandlung von Wurzelkaries, Füllungen von Klasse-III-Kavitäten, Restauration von Milchzähnen, als Unterfüllung bzw. Liner, für den Stumpfaufbau sowie für temporäre Füllungen.

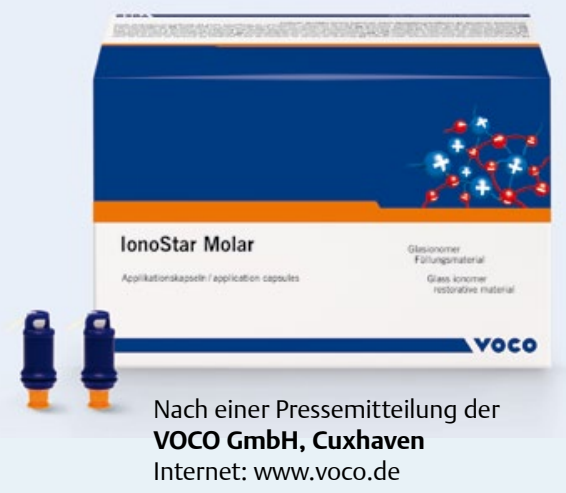

Internet: www.voco.de

\section{Chirurgie \\ Neues Kit für Einsteiger}

BIOMET3i bietet Zahnärzten mit einem neuen kompakten Chirurgie-Kit einen guten Einstieg in die Implantologie. Das Slim Chirurgie-Kit für zahnwurzelförmige Implantate ist ein Tray mit allen Instrumenten, die der Zahnarzt für das Setzen von wurzelförmigen Implantaten mit Durchmessern von 3,25 und $4 \mathrm{~mm}$ sowie Längen von $8,5-13 \mathrm{~mm}$ benötigt. Das neue Kit ist in 2 Insertionsausführungen erhältlich: für Implantate mit Certain-Verbindung und Außensechskant- (SLIMKTE) oder nur für die Certain-Verbindung (SLIMKT).
Durch die Reduktion auf die wesentlichen Bauteile wird es besonders Einsteigern ermöglicht, die häufig doch komplexen Arbeitsschritte in der zahnärztlichen Implantologie sicher zu meistern. Mit der klaren Kodierung entsprechend der benötigten Bohrerprotokolle und den passenden Instrumenten in Griffnähe unterstützt das Kit den Zahnarzt bei einem sicheren und effizienten Workflow.

Neukunden macht BIOMET3i ein besonderes Angebot: sie erhalten beim Kauf von 10 T3-Implantaten ein Slim Chirur-

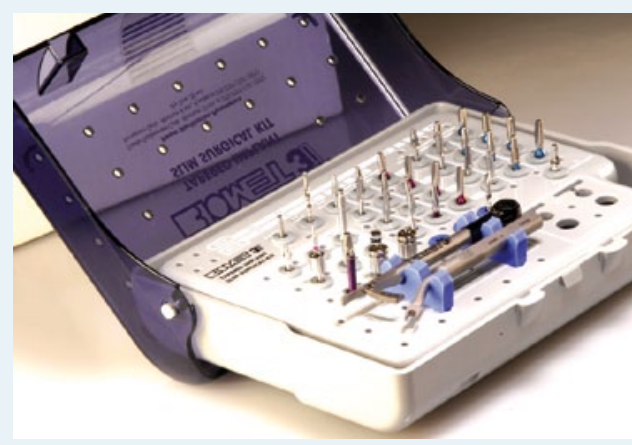

gie-Kit (SLIMKT) und ein prothetisches Starter-Kit gratis dazu.

Nach einer Pressemitteilung der BIOMET 3i Deutschland GmbH, München

\section{Hygiene}

\section{Farben für mehr Sicherheit}

Einfach, wirkungsvoll und übersichtlich ist die System-Hygiene von Dürr Dental für optimalen Infektionsschutz. Um Anwendern mehr Sicherheit bei der systematische Instrumentenaufbereitung zu geben, hat Dürr Dental seine Präparate daher mit einem speziellen 4-Farbsystem gekennzeichnet. Über 30 Produkte werden darin ihren Einsatzbereichen klar und übersichtlich zugeordnet: grün für Flächen, blau für Instrumente, gelb für Spezialbereiche und Sauganlagen und rosa für Haut und Hände. Das Dürr Dental-Konzentrat zur Instrumentendesinfektion ID 213 oder die BohrerDesinfektion ID 220 sind mit „blau“ gekennzeichnet. Beide Präparate sind bakterizid, tuberkulozid, fungizid und voll viruzid gegenüber behüllten und

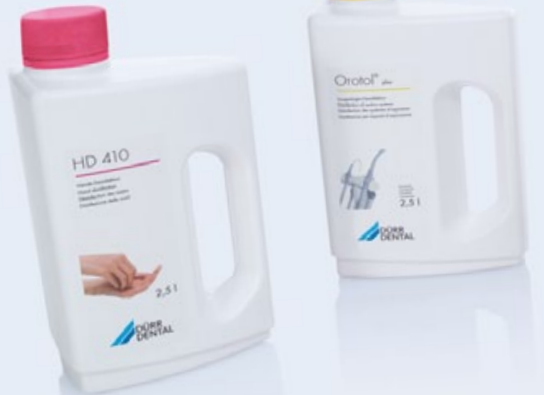

unbehüllten Viren und erfüllen alle Anforderungen des Robert-Koch-Instituts. Für die „rosa“ Händedesinfektion stehen Produkte wie das Einreibepräparat HD 410 zur Verfügung. Die Desinfektion kontaminierter Oberflächen ist „grün“ mit der Wischdesinfektion FD 322 oder der voll viruziden Schnelldesinfektion FD 333 (Einwirkzeit $1 \mathrm{~min}$ ). Desinfektions- und Reinigungsprodukte aus dem

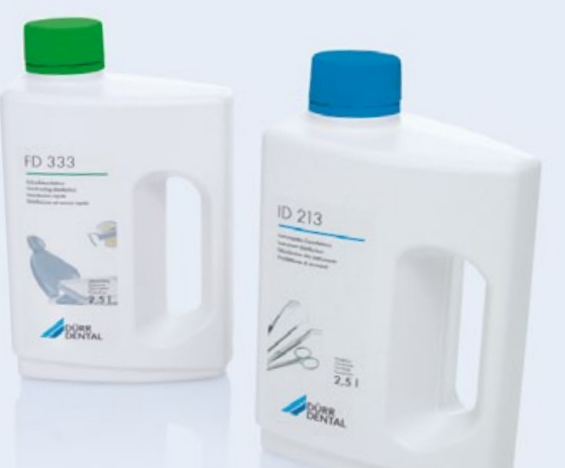

„gelb“ gekennzeichneten Spezialbereich sind für die Beseitigung pathogener Keime aus der Absauganlage zuständig. Ihre Wirkstoffe sind genau für diese Anwendung auf ihre Wirksamkeit hin überprüft worden.

Nach einer Pressemitteilung der Dürr Dental AG, Bietigheim-Bissingen

Internet: www.duerrdental.com 